\begin{tabular}{|c|l|}
\hline Title & Theoretical investigation of new quantum-cross structure device as a candidate bey ond CMOS \\
\hline Author(s) & Kondo, Kenji; Kaiju, Hideo; Ishibashi, A kira \\
\hline Citation & $\begin{array}{l}\text { MRS Proceedings, 1067, 1067-B03-01 } \\
\text { https://doi.org/L0.1557/PROC-1067-B03-01 }\end{array}$ \\
\hline Issue Date & 2008-06-11 \\
\hline Doc URL & http://hdl.handle.net/2115/57074 \\
\hline Rights & ○ 2008 Materials Research Society \\
\hline Type & article \\
\hline File Information & Theoretical Investigation of New Quantum-Cross Structure Device as a Candidate beyond CMOS.pdf \\
\hline
\end{tabular}

Instructions for use 


\title{
Theoretical Investigation of New Quantum-Cross-Structure Device as a Candidate beyond CMOS
}

Kenji Kondo, Hideo Kaiju, and Akira Ishibashi

Laboratory of Quantum Electronics, Research Institute for Electronic Science, Hokkaido

University, Sapporo, 060-0812, Japan

\begin{abstract}
We propose a new quantum cross structure (QCS) device as a candidate beyond CMOS. The QCS consists of two metal nano-ribbons having edge-to-edge configuration like crossed fins.The QCS has potential application in both switching devices and high-density memories by sandwiching a few molecules and atoms. The QCS can also have electrodes with different dimensional electron systems because we can change the widths, the lengths, and the heights of two metal nano-ribbons, respectively. Changing the dimensions of electron systems in both electrodes, we have calculated the current-voltage characteristics depending on the coupling constants between a molecule and the electrode. We find that the conductance peak is much sharper in case of weak coupling regardless of dimensions of electron systems in electrodes, compared to strong coupling case. We also find that the conductance peak of QCS having electrodes with two-dimensional electron systems (2DES) is much sharper than that of QCS having electrodes with three-dimensional electron systems (3DES) in case of strong coupling because of quantum size effect of 2DES. These results imply that the QCS with the very sharp conductance peak can serve as the devices to switch on and off by very small voltage change.
\end{abstract}

\section{INTRODUCTION}

Nowadays, many researchers have paid attention to post-silicon devices [1,2]. One of the several post-silicon devices is a cross-bar memory device based on molecular devices fabricated by nanoimprint lithography, which has achieved the production of 30-nm half-pitch patterning $[3,4]$. However, today's production procedures such as nanoimprint lithography, and optical lithography, and electron-beam lithography, do not allow for the resolution to achieve sub-20-nm line-width structures.

Recently, we have proposed a double nano-"baumkuchen" (DNB) structure, composed of two thin slices of alternating metal/insulator nano-"baumkuchen" as a lithography-free nanostructure fabrication technology [5,6], and a quantum cross structure device [7,8]. The schematic illustration of the fabrication procedure is shown in figure 1. First, the metal/insulator (organic film) spiral heterostructure is fabricated using a vacuum evaporator including a film-rolled-up system. Then, two thin slices of the metal/insulator nano-"baumkuchen" are cut out from the metal/insulator spiral heterostructure. Finally, the two thin slices are attached together face to face so that each stripe is crossing. The DNB has potential application in switching devices or high-density memories, the cross point of which can be scaled down to ultimate feature sizes of a few nanometers due to the film thickness determined by the metal-deposition rate, ranging from $0.01 \mathrm{~nm} / \mathrm{s}$ to $1 \mathrm{~nm} / \mathrm{s}$. We call one element of the DNB structure a QCS device that consists of two metal nano-ribbons having the edge-to-edge configuration shown in figure 1(c). The nanoribbon electrodes can have different dimensional electron systems, such as 2DES or 3DES with their widths, lengths, and heights changed. We have already calculated the quasi-particle energy 
spectrum in two-dimensional (2D) electrodes using GW approximation [7]. Sandwiching an atom or a molecule between the two metal ribbons, the QCS device can detect the essential information of individual atoms or molecules. Also, we can make tunneling devices by sandwiching insulators, such as self-assembled monolayers (SAMs), DNA, or metal oxides. Therefore, we have reported about the calculation of electronic transport of tunneling in QCS devices [8]. In this paper, we have formulated the theory of electronic transport, which can deal with the difference of dimensionality of electrons in electrodes within the framework of the Anderson Hamiltonian, and we have studied the current-voltage characteristics of QCS devices with application of the theory. It is shown that the QCS devices are promising ones beyond CMOS quantitatively.

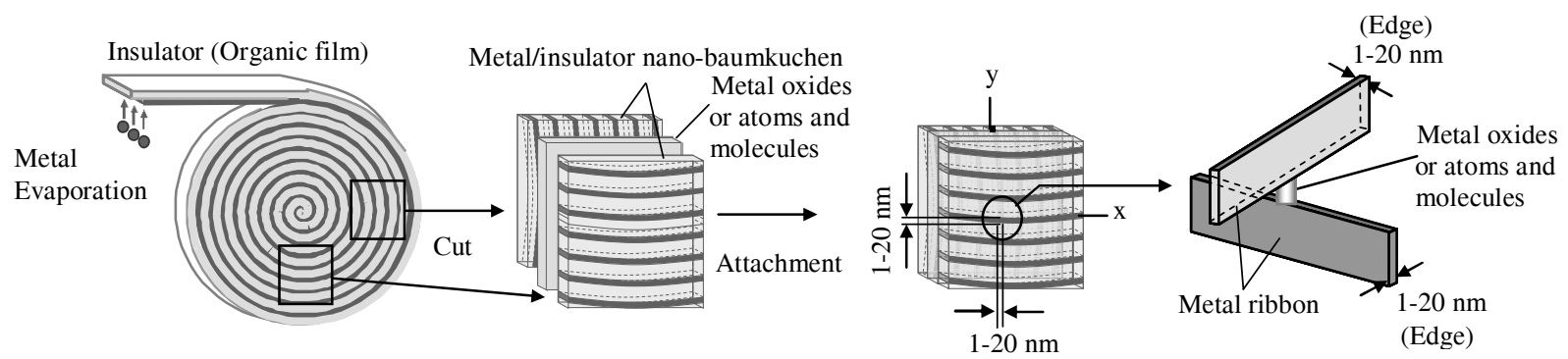

(a) Metal/insulator (organic film)

(b) Double nano-baumkuchen

(c) Quantum cross structure device spiral heterostructure by evaporation

by cut and attachment process

Figure 1. Schematic illustration of (a) a metal/insulator (organic film) spiral heterostructure, (b) a double nano-"baumkuchen" (DNB) structure, and (c) a quantum cross structure (QCS) device.

\section{THEORY}

We study the current-voltage characteristics of QCS devices with a molecule sandwiched between two metal electrodes. The molecule is assumed to have two energy levels.

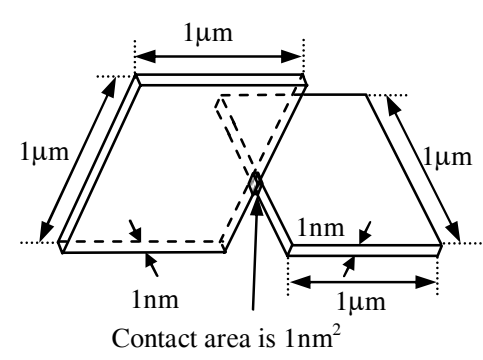

(a) QCS model with 2D electrodes

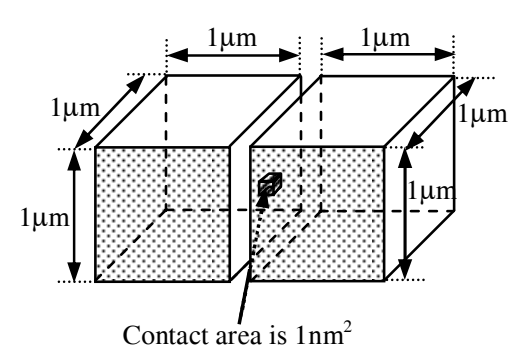

(b) QCS model with 3D electrodes

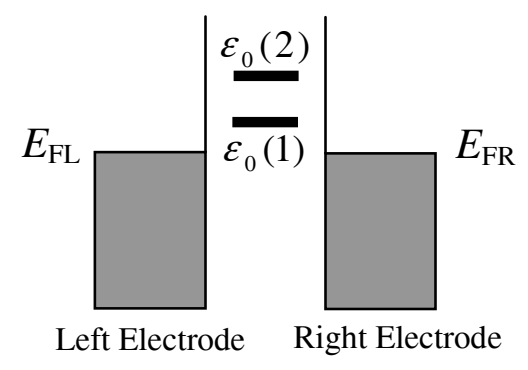

(c) Energy diagram for QCS devices

Figure 2. Schematic illustration of QCS device model (a) with 2D electrodes, and (b) with three-dimensional (3D) electrodes. The small shading box between the electrodes indicates a molecule position. (c) The energy diagram for QCS devices with 2D, 3D electrodes.

The models of QCS devices with 2D electrodes, 3D electrodes are shown in figure 2(a), figure 2(b), respectively. The energy diagram for the QCS device models is also shown in figure 2(c). We analyze the transport characteristics using the Anderson Hamiltonian, taking into 
consideration the dimensionality of electrons in both electrodes. In this case, the Anderson Hamiltonian is described as follows:

$$
H=H_{\text {Electrodes }}+H_{\text {mole }}+H_{\mathrm{t}}
$$

where

$$
\begin{aligned}
& H_{\text {Electrodes }}=\sum_{\alpha=L, R} \sum_{k, \sigma} \varepsilon_{\boldsymbol{k} \sigma} c_{\alpha, \boldsymbol{k} \sigma}^{+} c_{\alpha, \boldsymbol{k} \sigma}, \\
& H_{\text {mole }}=\sum_{i, \sigma} \varepsilon_{0}(i) a_{i, \sigma}^{+} a_{i, \sigma}, \\
& H_{\mathrm{t}}=\sum_{\alpha=L, R} \sum_{k, \sigma} \sum_{i, \sigma}\left(V_{\alpha} c_{\alpha, \boldsymbol{k} \sigma}^{+} a_{i, \sigma}+\text { h.c. }\right) .
\end{aligned}
$$

$H_{\text {Electrodes }}$ is the Hamiltonian of both metal electrodes, $\varepsilon_{k \sigma}=\frac{\hbar^{2} \boldsymbol{k}^{2}}{2 m}, m$ is the free electron mass, and $\hbar$ is the Planck's constant $h$ divided by $2 \pi$. The wave vector $\boldsymbol{k}$ is a $2 \mathrm{D}$ vector in $2 \mathrm{D}$ electrodes and a 3D vector in 3D electrodes. If you want to apply this theory to the interacting case, you need only replace $\varepsilon_{k \sigma}$ with quasi-particle energy spectrum obtained using GW approximation [7]. $c_{\alpha, k \sigma}^{+}$and $c_{\alpha, k \sigma}$ are creation and annihilation operators for electrons of wave vector $\boldsymbol{k}$ and spin index $\sigma$ in $\alpha$ electrode. $\alpha$ indicates the left or right electrode. The creation and annihilation operators obey the standard fermion anticommunication rules.

$$
\left[c_{\alpha, k \sigma}, c_{\alpha^{\prime}, k^{\prime} \sigma^{\prime}}^{+}\right]_{+}=\delta_{k, k^{\prime}} \delta_{\alpha, \alpha^{\prime}} \delta_{\sigma, \sigma^{\prime}}, \quad\left[c_{\alpha, k \sigma}, c_{\alpha^{\prime}, k^{\prime} \sigma^{\prime}}\right]_{+}=0
$$

$H_{\text {mole }}$ is the Hamiltonian of a molecule sandwiched between both electrodes, and $\varepsilon_{0}(i)$ represents the $i$-th energy level of eigen-states of the molecule as shown in figure 2(c). We assumed the molecule had only two energy levels, $\varepsilon_{0}(1)=0.5 \mathrm{eV}, \varepsilon_{0}(2)=1 \mathrm{eV}$, estimated from Fermi levels $E_{\mathrm{FL}}, E_{\mathrm{FR}}$ of each electrode. The Fermi levels $E_{\mathrm{FL}}, E_{\mathrm{FR}}$ of each electrode were assumed to be equal and we used the value, $5.51 \mathrm{eV}$, of the gold for the Fermi level. $a_{i, \sigma}^{+}$and $a_{i, \sigma}$ are creation and annihilation operators for electrons of spin index $\sigma$ in the $i$-th energy level. These operators also obey the standard fermion anticommunication rules like $c_{\alpha, k \sigma}^{+}, c_{\alpha, k \sigma}$.

$H_{\mathrm{t}}$ is the transfer Hamiltonian between the sandwiched molecule and each electrode, $V_{\alpha}$ is the transfer matrix between the molecule and $\alpha$ electrode. This value determines the coupling strength between each electrode and the molecule. Considering $H_{\mathrm{t}}$ as a perturbation, we have investigated the current-voltage characteristics from right to left electrode by changing these coupling strengths and the dimensions of electron systems in electrodes, using many-body perturbation technique. The electrons flow from left to right electrode. The transition probability $P_{L \rightarrow R}$ of electrons from left to right electrode is given by 


$$
P_{L \rightarrow R}=2 \sum_{\boldsymbol{k}} \sum_{\boldsymbol{k}^{\prime}} \frac{2 \pi}{\hbar}\left|<R \boldsymbol{k}^{\prime}\right| T|L \boldsymbol{k}>|^{2} \delta\left(\varepsilon_{R \boldsymbol{k}^{\prime}}-\varepsilon_{L \boldsymbol{k}}\right) f\left(\varepsilon_{L \boldsymbol{k}}-E_{\mathrm{FL}}\right)\left[1-f\left(\varepsilon_{R \boldsymbol{k}^{\prime}}-E_{\mathrm{FR}}\right)\right] \text {, }
$$

where $e$ is the elementary charge, $f(\varepsilon)$ is the Fermi-Dirac distribution function, $|R \boldsymbol{k}\rangle, \mid L \boldsymbol{k}>$ are Bloch functions of electrons with eigen-energies $\varepsilon_{R k}, \varepsilon_{L k}$ in right, left electrode, respectively, and $T$ is so-called $T$-matrix, which is given in the following:

$$
T=H_{\mathrm{t}}+H_{\mathrm{t}} G_{0} T, \quad G_{0}=\frac{1}{\varepsilon-H_{0}+i \delta}, \quad H_{0}=H_{\text {Electrodes }}+H_{\text {mole }},
$$

where $G_{0}$ is the bare Green's function, and $\delta$ is the infinitesimal positive quantity. After tedious manipulation, the current $I$ from right to left electrode is as follows:

$$
\begin{aligned}
I & =e\left(P_{L \rightarrow R}-P_{R \rightarrow L}\right) \\
& =\frac{2 e^{2}}{h} \int_{E_{\mathrm{FR}}}^{E_{\mathrm{FR}}+e V} d \varepsilon \sum_{i}\left(\frac{4 \Gamma_{\mathrm{L}}(\varepsilon) \Gamma_{\mathrm{R}}(\varepsilon)}{\left(\varepsilon-\varepsilon_{0}(i)\right)^{2}+(\Gamma(\varepsilon))^{2}}\right)\left[f\left(\varepsilon-e V-E_{\mathrm{FR}}\right)-f\left(\varepsilon-E_{\mathrm{FR}}\right)\right],
\end{aligned}
$$

where $\Gamma(\varepsilon)$ is the sum of $\Gamma_{\mathrm{L}}(\varepsilon)$ and $\Gamma_{\mathrm{R}}(\varepsilon)$, and $\Gamma_{\mathrm{L}}(\varepsilon), \Gamma_{\mathrm{R}}(\varepsilon)$ are coupling strengths between the molecule and left, right electrode, respectively, which are given in the following:

$$
\Gamma_{\mathrm{L}}(\varepsilon)=\pi D_{\mathrm{L}}(\varepsilon)\left|V_{\mathrm{L}}\right|^{2}, \quad \Gamma_{\mathrm{R}}(\varepsilon)=\pi D_{\mathrm{R}}(\varepsilon)\left|V_{\mathrm{R}}\right|^{2}
$$

where $D_{\mathrm{L}}(\varepsilon), D_{\mathrm{R}}(\varepsilon)$ are densities of states of electrons in left, right electrode, respectively. These coupling strengths correspond to the broadening of the energy level of the sandwiched molecule induced by the interaction with itinerant electrons in each electrode.

\section{RESULTS and DISCUSSION}

We have calculated the transport characteristics at room temperature $300 \mathrm{~K}$. Figure 3(a), 3(b) show the $I-V$ characteristics of QCS devices with 2D electrodes, 3D electrodes, respectively, under the weak coupling condition. We regard the coupling as the weak coupling when the energy of coupling strength is smaller than that of the ambient temperature $27 \mathrm{meV}$. In this situation, the coupling constants $V_{\mathrm{L}}, V_{\mathrm{R}}$ are $0.2 \mathrm{meV}$, corresponding to the coupling strengths $\Gamma_{\mathrm{L}}(\varepsilon)=\Gamma_{\mathrm{R}}(\varepsilon)=1.57 \mathrm{meV}$. We notice that both the results show the sharp steps at the positions of the energy level of the sandwiched molecule. Even at room temperature, these conductance peaks are sharp regardless of dimensionality of electrodes. The results are attributed to the discreteness of the energy level of the molecule. We can use a QCS device with 2D, or 3D electrodes as a switching device that works at very small voltage change under the weak coupling condition, which can become free from the so-called short channel effect of CMOS devices. Also, the flowing current of $0.5 \sim 1.0 \mu \mathrm{A}$ is very small. Therefore, the power consumption is in the order of $10 \sim 100 \mathrm{nW}$ at the device operation. The power consumption can be reduced until the much lower level by adjusting the coupling constants. 

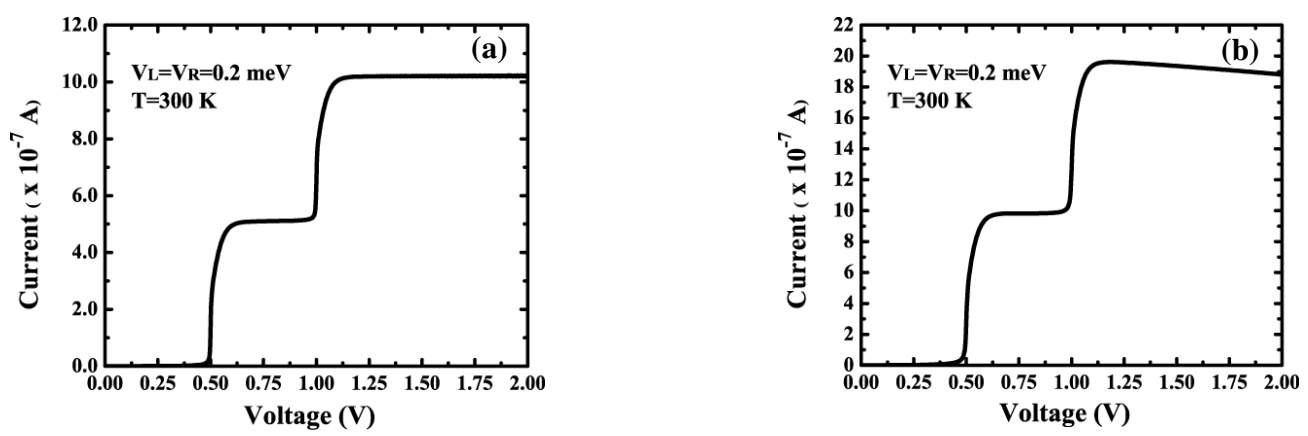

Figure 3. The current-voltage characteristics of QCS devices (a) with 2D electrodes, and (b) with 3D electrodes, under the weak coupling condition.

Figure 4(a), 4(b) show the $I-V$ characteristics of QCS devices with 2D electrodes, 3D electrodes, respectively, under the strong coupling condition. We regard the coupling as the strong coupling when the energy of coupling strength is larger than that of the ambient temperature. In this situation, the coupling constants $V_{\mathrm{L}}, V_{\mathrm{R}}$ are $1.0 \mathrm{meV}$, corresponding to the coupling strengths $\Gamma_{\mathrm{L}}(\varepsilon)=\Gamma_{\mathrm{R}}(\varepsilon)=39.27 \mathrm{meV}$. Compared to the weak coupling, the conductance peaks are smooth under the strong coupling condition for both QCS devices with 2D, 3D electrodes. However, the QCS device with 2D electrodes has much sharper peaks than one with 3D electrodes. The QCS device with 2D electrodes can still work as a switching device. This difference is attributed to 2D density of states changed due to the quantization of the energy of 2DES. As shown in figure 3,4, we find that the current of QCS device with 3D electrodes is larger than that of QCS device with 2D electrodes. This result is attributed to the larger density of states of 3D electrodes than that of 2D electrodes.
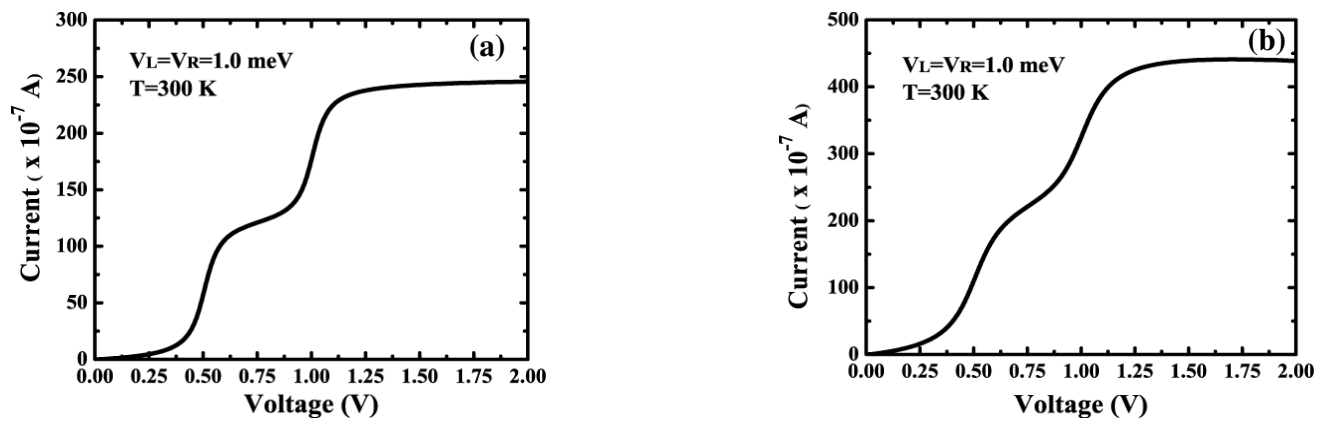

Figure 4. The current-voltage characteristics of QCS devices (a) with 2D electrodes, and (b) with $3 \mathrm{D}$ electrodes, under the strong coupling condition.

Finally, we consider the strong coupling limit. The strong coupling limit means that the energy of the coupling strengths $\Gamma_{\mathrm{L}}(\varepsilon), \Gamma_{\mathrm{R}}(\varepsilon)$ are much larger than that of the ambient temperature. In this situation, we used the coupling constants $V_{\mathrm{L}}, V_{\mathrm{R}}$ of $10.0 \mathrm{meV}$, corresponding to the coupling strengths $\Gamma_{\mathrm{L}}(\varepsilon)=\Gamma_{\mathrm{R}}(\varepsilon)=3927 \mathrm{meV}$. The energy level of the sandwiched molecule is almost continuous because of the wide broadening induced by the strong coupling with the itinerant electrons. Therefore, we expect the $I-V$ characteristics like the ohmic contact without the molecule. The $I-V$ characteristics of QCS devices under the strong coupling limit are shown in figure 5(a), (b). As expected, we can obtain the ohmic-like $I$ - $V$ characteristics, justifying the theory we have developed. To our knowledge, this is the first time to show 
explicitly that the $I$ - $V$ curve reduces to the ohmic-like one under the strong coupling limit within the framework of the Anderson Hamiltonian.
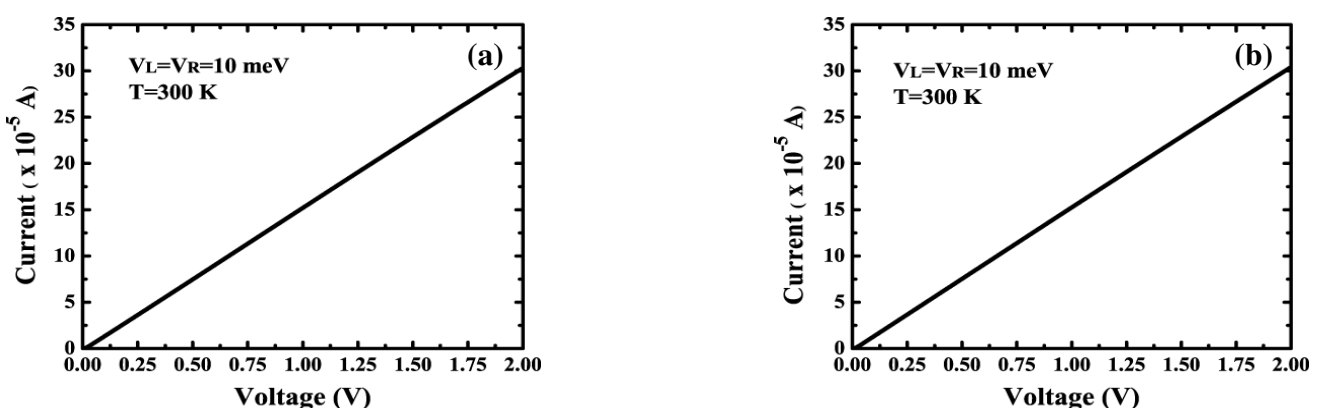

Figure 5. The current-voltage characteristics of QCS devices (a) with $2 \mathrm{D}$ electrodes, and (b) with $3 \mathrm{D}$ electrodes, under the strong coupling limit.

\section{CONCLUSIONS}

We have formulated the electronic transport theory, dealing with the difference of dimensionality of electrons in electrodes within the framework of the Anderson Hamiltonian. We have studied the $I-V$ characteristics of QCS devices using the theory. We find that the QCS devices with 2D, 3D electrodes have sharp conductance peaks under the weak coupling condition even at room temperature. Therefore, we can expect the QCS device with 2D, or 3D electrodes to be the switching device that works at very small voltage change with the small current. The QCS device can operate at very small power consumption of $10 \sim 100 \mathrm{nW}$. These results imply that the QCS device is a promising one beyond CMOS. We consider that it is novel that this new QCS device is shown to be available for a switching device quantitatively, and that the theory is shown to easily apply to the interacting case.

\section{ACKNOWLEDGMENTS}

This research has been partially supported by Special Education and Research Expenses from Post-Silicon Materials and Devices Research Alliance.

\section{REFERENCES}

1. J. Chen, M. A. Reed, A. M. Rawlett, and J. M. Tour, Science 286, 1550(1999).

2. Semiconductor Industry Association, International Technology Roadmap for Semiconductors, 2005 ed.

3. W. Wu, G.-Y. Jung, D. L. Olynick, J. Straznicky, Z. Li, X. Li, D. A. A. Ohlberg, Y. Chen, S. -Y. Wang, J. A. Liddle, W. M. Tong, R. S. Williams, Appl. Phys. A 80, 1173 (2005).

4. G. Y. Jung, W. Wu, S. Ganapathiappan, D. A. A. Ohlberg, M. Saifislam, X. Li, D. L. Olynick, H. Lee, Y. Chen, S. Y. Wang, W. M. Tong, R. S. Williams, Appl. Phys. A 81, 1331 (2005).

5. A. Ishibashi, Proc. Int. Symp. on Nano Science and Technology, 44 (2004).

6. H. Kaiju, A. Ono, N. Kawaguchi, and A. Ishibashi, Jpn. J. Appl. Phys. 47, 244 (2008).

7. K. Kondo and A. Ishibashi: Jpn. J. Appl. Phys. 45, 9137 (2006).

8. H. Kaiju, K. Kondo, and A. Ishibashi: Mater. Res. Soc. Symp. Proc. 961, O5.5.1 (2007). 